\title{
TRAUMA CARDÍACO: ESTUDO DE NECROPSIAS
}

\section{CARDIAC TRAUMA: AUTOPSY FINDINGS}

\author{
Gustavo Pereira Fraga, TCBC-SPㅜ; Luiz Rogério Heinzl²; Bárbara Sugui Longhi²; \\ Daniel Carlos da Silva ${ }^{3}$; Francisco Américo Fernandes Neto, TCBC-SP ${ }^{4}$; Mario Mantovani, TCBC-SP
}

\begin{abstract}
RESUMO: Objetivo: Pacientes vítimas de trauma cardíaco morrem, na maioria das vezes, antes de receberem atendimento médico. Porém, são poucos os estudos epidemiológicos deste tipo de lesão descrevendo a porcentagem de pacientes que chegam a ser tratados. O objetivo do presente trabalho é avaliar as características das vítimas de trauma cardíaco através da interpretação de laudos de necropsia. Método: Foram revisados 1.976 casos de óbito por causas externas submetidos a necropsia no Instituto Médico Legal de Campinas, num período de dois anos. Os casos foram divididos em dois grupos: I, trauma penetrante; e II, trauma fechado. Resultados: Houve predomínio dos traumas penetrantes (1.294 casos - 65,5\%). Trauma cardíaco foi identificado em 359 laudos (18,2\%), sendo 296 do grupo I e 63 do grupo II. No grupo I, 73,6\% dos óbitos ocorreram no local do trauma e apenas 18 pacientes (6\%) foram atendidos em hospital e submetidos a toracotomia. No grupo II o tratamento cirúrgico foi indicado em dois dos 14 traumatizados (3,2\% dos traumas fechados) admitidos com presença de sinais vitais. A câmara cardíaca mais acometida no grupo I foi o ventrículo esquerdo (lesão isolada em $24,6 \%$ dos casos) e no grupo II o ventrículo direito (25\%). Conclusão: Conclui-se que as lesões cardíacas são eminentemente fatais e apenas 5,6\% destes traumatizados que morreram chegaram a receber tratamento efetivo (Rev. Col. Bras. Cir. 2004; 31(6): 386-390).
\end{abstract}

Descritores: Traumatismos cardíacos; Coração; Autopsia; Traumatismos torácicos.

\section{INTRODUÇÃO}

As descrições de lesões cardíacas são conhecidas há milênios. Desde o Papiro de Edwin Smith, em 3000 a.C., um dos primeiros relatos deste tipo de lesão, até o final do século XIX, pouco se acrescentou no tratamento efetivo do trauma cardíaco ${ }^{1}$. O tratamento passou da observação clínica, em 1800, até a agressiva terapia operatória na virada do século. Observa-se uma tendência mundial em aumentar a sobrevida geral do traumatismo cardíaco devido a existência de serviços cada vez mais treinados, tanto no atendimento pré-hospitalar quanto no hospitalar, altos índices de suspeição clínica e indicação de toracotomia de emergên$\mathrm{cia}^{2}$. Contudo, em virtude do crescimento exponencial do trauma, em decorrência da violência urbana e dos acidentes envolvendo veículos automotores, tornam-se substancialmente graves as lesões cardíacas, freqüentemente provocando sangramento profuso e exacerbando as casuísticas de estudos de necropsia ${ }^{2-7}$. Estes estudos tornam-se, portanto, essenciais para avaliar a gravidade das lesões, bem como evidenciar as áreas que estão inadequadamente servidas pelo sistema pré-hospitalar5 ${ }^{5}$. Assim, procurou-se individualizar as características dos traumas cardíacos que ocorreram num período de 24 meses, através da análise dos laudos de necropsia do Instituto Médico Legal (IML) de Campinas.

\section{MÉTODO}

Foram analisados 1.976 laudos de necropsia por causas externas realizadas no IML de Campinas, no período de janeiro de 2000 a dezembro de 2001. Os laudos foram transcritos e analisados no sistema de base de dados EPI-INFO, versão 6.04, contendo as seguintes informações: identificação, gênero, idade, data do óbito, mecanismo de trauma, descrição de lesões externas e de órgãos internos, atendimento préhospitalar e/ou médico, acessos cirúrgicos realizados, intervalo de tempo entre o trauma e o óbito, local e causa do óbito. Conforme o mecanismo de trauma, os laudos em que havia descrição de trauma cardíaco foram separados em dois grupos para estudo. O grupo I foi composto pelas vítimas de trauma penetrante, divididos em ferimento por projétil de arma de fogo (FPAF), ferimento por arma branca ou outros objetos pérfuro-cortantes (FAB), ou ainda, a associação destes diferentes mecanismos. O grupo II incluiu laudos de vítimas de traumatismo contuso ou fechado (TF): eventos automobilísticos, motociclísticos, atropelamento, queda, espancamento e outros. A associação dos mecanismos foi separada e classificada de acordo com a importância para a lesão cardíaca (TF associado com FAB, TF associado com FPAF, ou apenas a associação FPAF com FAB).

Foram consideradas como lesão cardíaca a presença de lesões penetrantes do pericárdio, ou câmaras cardíacas ou

1. Professor Assistente Doutor da Disciplina de Cirurgia do Trauma da FCM-Unicamp e do Departamento de Cirurgia do Hospital Municipal “Dr. Mário Gatti”, Campinas-SP

2. Aluno(a) de Graduação e da Liga do Trauma da FCM PUC-Campinas

3. Aluno de Graduação e da Liga do Trauma da FCM-Unicamp

4. Mestre em Cirurgia; Coordenador do Curso de Medicina Legal da FCM-Unicamp e Médico Assistente do Departamento de Cirurgia do Hospital Municipal “Dr. Mário Gatti”, Campinas-SP

5. Professor Titular e Chefe da Disciplina de Cirurgia do Trauma da FCM-Unicamp

Recebido em 25/05/2004

Aceito para publicação em 03/08/2004

Trabalho realizado no Instituto Médico Legal (IML) de Campinas, SP. 
Tabela 1 - Número de laudos e porcentagem conforme o mecanismo de trauma e a presença de lesões de coração.

\begin{tabular}{llrrrr}
\hline Grupo & Mecanismo de trauma* & Necropsias & \multicolumn{3}{c}{ Traumatismo cardíaco } \\
& & $\mathbf{n}$ & $\mathbf{0}$ & $\mathbf{n}$ & $\mathbf{\%}$ \\
\hline \multirow{2}{*}{$\mathbf{( n = 2 9 6 )}$} & 1.179 & 59,7 & 260 & 22,0 \\
& FPAF & 75 & 3,8 & 25 & 33,3 \\
& FAB & 15 & 0,7 & 5 & 33,3 \\
& FPAF + FAB & 7 & 0,3 & 2 & 28,5 \\
& FAB + TF & 18 & 0,9 & 4 & 22,2 \\
II & FPAF + TF & 184 & 9,3 & 35 & 19,0 \\
$(\mathbf{n = 6 3 )}$ & Acidente de carro & 47 & 2,4 & 5 & 10,6 \\
& Acidente de moto & 156 & 7,9 & 17 & 10,9 \\
& Atropelamento & 81 & 4,1 & 5 & 6,2 \\
& Quedas & 52 & 2,6 & - & - \\
& Espancamento & 162 & 8,2 & 1 & 0,6 \\
\hline & Outros & 1.976 & \multicolumn{2}{c}{18,2} \\
\hline
\end{tabular}

* FPAF = ferimento por projétil de arma de fogo; FAB = ferimento por arma branca; $T F=$ trauma fechado.

vasos da base. Os grandes hematomas ou lacerações do miocárdio ou troncos vasculares do coração também foram incluídos na casuística.

\section{RESULTADOS}

Em 359 casos (18,2\% dos laudos) foi descrito trauma cardíaco. No grupo I foram incluídos 296 casos, com incidência de lesão cardíaca de 22,9\% nos 1.294 laudos de trauma penetrante. No grupo II foi identificado lesão de coração em 63 casos, correspondendo a 9,2\% dos 682 laudos de trauma fechado (Tabela 1).

Considerando os 1.976 laudos, em 986 (49,9\%) havia descrição de lesões torácicas, como orifícios de entrada provocados por FPAF, lesões pérfuro-cortantes por FAB, fraturas de costelas, hematomas ou escoriações da parede torácica em vítimas de TF. Dos traumas torácicos, houve lesão cardíaca em 329 casos. Entretanto, em 30 necropsias (1,5\% do total de laudos) foram encontradas lesões no coração sem a presença de qualquer traumatismo de tórax, sendo 20 casos de trauma penetrante com FPAF localizado em outro segmento corpóreo, e 10 por traumatismo contuso.

Em relação ao Grupo I (n=296), houve predomínio do sexo masculino (278 casos - 93,9\%) sobre o feminino (18 $6,1 \%)$. A faixa etária mais acometida foi entre 20 e 29 anos (45,7\%), sendo a média de idade de 27,9 anos (variação de um a 68 anos).

No grupo I, os FPAF foram os responsáveis pelo maior número de lesões cardíacas, com 260 casos (87,8\% das lesões por trauma penetrante). O número médio de FPAF encontrados nas vítimas foi de 5,71 (variando de um a 30). As lesões associadas mais freqüentes foram: crânio (96 casos 36,9\%), fígado ( 87 - 33,5\%), diafragma (38 - 14,6\%), extremidades (28 - 10,8\%) e baço (18 - 6,9\%). Os FAB, isoladamente, ocorreram em 25 casos (8,4\%) e tiveram média de 7,54 lesões perfurantes nos diferentes segmentos corpóreos por vítima (variando de uma 18). A maioria das lesões associadas, provocadas por este mecanismo, foi encontrada no fígado (12 casos - 48,0\%), nos vasos torácicos (cinco - 20,0\%) e no crânio (cinco - 20,0\%). Houve associação dos dois mecanismos em cinco casos $(1,4 \%)$ e em seis $(1,8 \%)$ havia um instrumento perfurante somado a um contundente (Tabela 1).

Ainda no grupo I, os óbitos imediatos (na cena do trauma) prevaleceram (218 casos - 73,6\%), e 78 (26,4\%) pacientes receberam atendimento pré-hospitalar, chegando 39 (13,2\%) com sinais vitais nos serviços hospitalares (Figura 1). Nestes serviços, houve intervenção cirúrgica em 18 casos (50\% dos admitidos em serviço hospitalar), sendo realizado acesso cirúrgico através de toracotomia ântero-lateral esquerda em 16 casos e toracofrenolaparotomia em dois pacientes. Nos pacientes tratados cirurgicamente, o período de sobrevida foi menor que 24 horas em 14 casos $(77,8 \%)$ e de um a sete dias em quatro pacientes (22,2\%), sendo a causa de morte as complicações do choque hemorrágico (três pacientes) e trauma raquimedular em um paciente. Em 122 laudos (41,2\%), havia

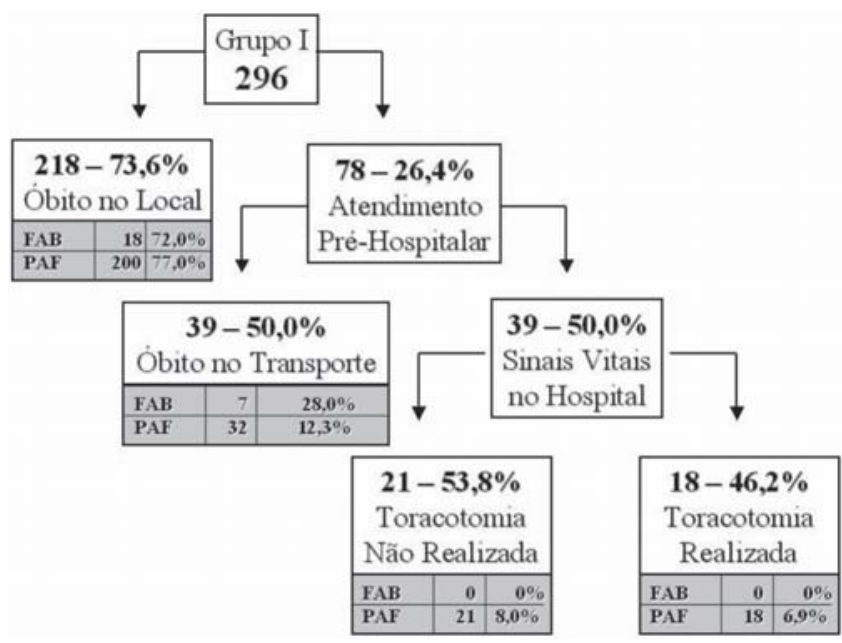

Figura 1 - Algoritmo conforme o atendimento e o momento do óbito nas vítimas do grupo $I$. 
descrições sobre a anatomia da lesão (perfuração de átrio, ventrículo ou vasos da base), conforme apresentado na Tabela 2.

Quanto ao Grupo II (n=63), o gênero masculino (47 $74,6 \%$ ) se sobrepôs ao feminino (16 - 25,4\%). A média de idade foi de 35 anos (variação de um a 79 anos) e a maioria se encontrava na faixa etária de 20 a 29 anos (23,8\%), seguida de 50 a 59 anos (20,6\%).

Os eventos automobilísticos foram os responsáveis pelo maior número de óbitos (35 - 55,5\% dos TF), posteriormente vindo o atropelamento (17 - 27,0\%) e os acidentes de motocicleta (cinco - 8,0\%) - Tabela 1. Trinta e sete traumatizados (58,7\%) morreram no local e 26 (41,3\%) receberam atendimento pré-hospitalar, chegando 14 (53,9\% das vítimas atendidas) com sinais de vida nos serviços hospitalares da cidade. Destes, em somente dois (14,3\%) foi realizado toracotomia (Figura $2)$. Dez pacientes $(71,4 \%)$ foram a óbito nas primeiras 24 horas e quatro $(28,6 \%)$ morreram com mais de sete dias, decorrente de complicações do choque hemorrágico (dois pacientes) e infecção associada a insuficiência renal (dois pacientes).

No grupo II, as lesões associadas ao trauma cardíaco mais freqüentes foram: crânio (37 - 58,7\%), fígado (37 - 58,7\%) e baço (29 - 46,0\%), ocorrendo mais de uma lesão na maioria dos pacientes. Em 28 laudos (44,4\%) havia descrição da lesão anatômica cardíaca provocada pelo trauma contuso (Tabela 2), com predomínio de lesões em vasos da base (13 casos 46,4\%).

\section{DISCUSSÃO}

O traumatismo torácico foi freqüente na amostra de 1.976 casos (986 - 49,9\%). Em 359 casos (18,2\% dos laudos) havia lesão no coração, uma incidência bem superior ao de Kulshrestha et al. ${ }^{6}$ que encontraram 8,2\% de ferimentos cardíacos nos traumas de tórax em necropsias de pacientes atendidos em seu serviço hospitalar, demonstrando que a maioria dos pacientes com trauma cardíaco penetrante morre antes que qualquer suporte avançado de vida possa ser oferecido ${ }^{8}$. Vinte laudos onde não havia qualquer descrição sobre trauma torácico penetrante, mas com lesão cardíaca, podem ser explicados pela trajetória do projétil que perfurou o abdome superior e o dorso, atingindo o coração, sendo isto descrito em casuísticas de pacientes tratados com lesão cardíaca ${ }^{9}$. Nos traumas contusos, em 10 laudos não havia sinais externos (hematomas, escoriações ou fraturas) no tórax, mas sabe-se que a desaceleração brusca pode tanto lacerar a base da aorta quando promover a ruptura de câmaras cardíacas, mesmo sendo o impacto lateral ${ }^{10}$.

Em relação ao gênero e à faixa etária, há pouca diferença entre os grupos I e II, sendo coincidentes com a literatu$\mathrm{ra}^{6}$. É relevante a variação da média de idade de 27,9 anos para o grupo I e 35 para o grupo II.

No grupo I, os FPAF perfazem quase a totalidade dos óbitos e são os responsáveis pelo grande número de traumas torácico e cardíaco em nosso meio. Os FPAF são potencialmente fatais devido à alta velocidade dos projéteis ${ }^{8}$. Assim, em casuísticas de atendimento hospitalar, os traumas cardía-
Tabela 2 - Lesões anatômicas do coração divididas conforme o grupo.

\begin{tabular}{lcccc}
\hline & \multicolumn{2}{c}{ Grupo I (n=122) } & \multicolumn{2}{c}{ Grupo II (n=28) } \\
& $\mathbf{n}$ & $\mathbf{\%}$ & $\mathbf{n}$ & $\mathbf{\%}$ \\
\hline Átrio Direito & 6 & 4,9 & 3 & 10,7 \\
Átrio Esquerdo & 1 & 0,8 & - & - \\
Ventrículo Direito & 18 & 14,8 & 7 & 25,0 \\
Ventrículo Esquerdo & 30 & 24,6 & 3 & 10,7 \\
Vasos da Base & 43 & 35,2 & 13 & 46,4 \\
Assoc. Câmaras & 24 & 19,7 & 2 & 7,2 \\
\hline
\end{tabular}

cos por FPAF têm as mais baixas taxas de sobrevida, porém representam a maioria dos caos em estudos de necropsia ${ }^{3,8}$. De uma maneira geral, as vítimas de FPAF têm prejuízo fisiológico severo, caracterizado pelo RTS (Revised Trauma Scale) e PI (Physiologic Index) mais baixos do que em vítimas de FAB, uma vez que as lesões por FPAF causam grandes lacerações nas câmaras, provocando sangramento constante que não pode ser contido por um saco pericárdico aberto, levando rapidamente ao choque hemorrágico ${ }^{2,11-13}$. Nos ferimentos cardíacos há duas distintas entidades clínicas, o choque hemorrágico e o tamponamento cardíaco, sendo este último mais comum em FAB. O tamponamento é um determinante crítico para a sobrevivência com o presuntivo benefício de prevenir a exsangüinação. A vantagem pode ser resultado do tempo que se leva para descomprimir o pericárdio, enquanto que simultaneamente controla-se o sangramento ${ }^{12,14}$. Assim, é evidente que a maioria das casuísticas hospitalares é constituída por vítimas de $\mathrm{FAB}^{2,4,8,12-15}$. Outro fator que deprime as chances de sobrevida é o elevado número de projéteis (média de 5,7 por necropsia) e de lesões pérfuro-cortantes (média de 7,54 por necropsia). Isto resulta em grandes lacerações do coração e elevada incidência de lesões associados, consequentemente levando à exsangüinação (193 casos $65,2 \%$ ) e à morte imediata, sem qualquer perspectiva de atendimento pré-hospitalar, como descrito em outros estudos ${ }^{6}$.

Os eventos automobilísticos foram os que mais fizeram vítimas de trauma cardíaco no grupo II (35 casos - 55,6\%).

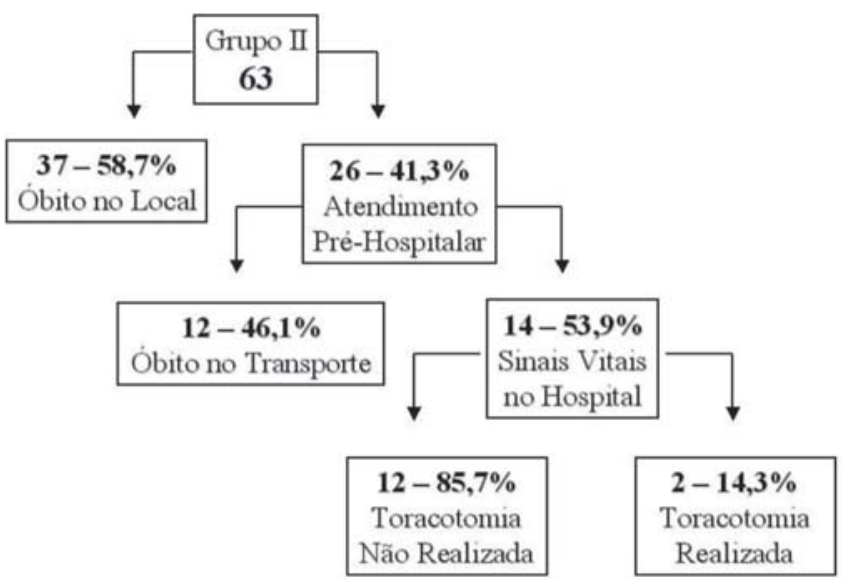

Figura 2 - Algoritmo conforme o atendimento e o momento do óbito nas vítimas do grupo II. 
A intensidade das contusões é refletida pelo elevado número de lesões associadas, principalmente no crânio e no fígado (37 casos - 58,7\%, respectivamente; lembrando que pode haver mais de uma estrutura lesada), o que torna o prognóstico do trauma contuso sombrio, mesmo com a maior eficiência do serviço de atendimento pré-hospitalar (41,3\% receberam tal atendimento). Daqueles que conseguiram chegar com sinais de vida nos hospitais (14 - 22,2\%), 71,4\% morreram nas primeiras 24 horas, ou por politraumatismo (que inclui TCE e choque hemorrágico) ou por exsangüinação. Nestes casos de trauma contuso, a toracotomia de reanimação parece ser ineficaz ${ }^{16}$.

Os vasos da base foram as estruturas mais lesadas nos grupos I (35,2\%) e II (46,4\%), sendo englobados a aorta ascendente, artérias pulmonares, veias pulmonares e a veia cava superior. Burkhart et al. ${ }^{10}$ relataram lesão de aorta ascendente em 12,4\% de 1.957 necropsias por trauma. Em 84,7\% dos casos, a lesão estava relacionada a eventos automobilísticos. O local mais comum de lesão foi o istmo da artéria $(58 \%)^{10}$. A maioria dos óbitos em que havia lesão dos vasos da base, a laceração da aorta era prevalente ${ }^{9,10}$. Kulshrestha et al. ${ }^{6}$ notaram que o ventrículo esquerdo (VE) tende a ser mais envolvido no TF do que o ventrículo direito (VD). A alta pressão sistólica intracavitária e a baixa complacência do VE favorecem a ruptura desta câmara por TF, contudo, a contusão miocárdica é comum no VD, o que pode ter sido significante na amostra ${ }^{6}$.

Considerando apenas as câmaras cardíacas lesadas, o VE prevaleceu no grupo I (24,6\%), coincidindo com os dados de Durham et al. ${ }^{16}$ e contrastando com os de Kulshrestha et al. ${ }^{6}$ e os de Campbell et al. ${ }^{5}$, onde o VD foi o mais atingido nos traumatismos penetrantes. A baixa incidência das lesões atriais em ambos os grupos é facilmente explicada pela proteção de sua posição posterior .

Dependendo do serviço pré-hospitalar, cerca de 10 a $30 \%$ das vítimas chegam ao hospital ${ }^{8}$. Cerca de 14,8\% das vítimas (englobando os dois grupos) de trauma cardíaco chegaram a receber atendimento hospitalar em Campinas. É evi- dente que o grande número de lesões associadas, bem como o alto índice de ferimentos ventriculares e dos vasos da base (em ambos os grupos) corroboram com o elevado número de pacientes que morrem no transporte ou que chegam sem sinais de vida ao hospital ${ }^{5}$. Entretanto, quanto mais rápido e mais eficiente for o serviço pré-hospitalar, maior o número de vítimas em estado agônico é esperado nos centros de referência em trauma, diminuindo o tempo para a intervenção cirúrgica, que é provavelmente o fator mais importante para a sobrevida ${ }^{5,16}$.

Com o presente estudo pode-se concluir que as lesões cardíacas são eminentemente fatais e apenas 5,6\% dos traumatizados que morreram chegaram a receber tratamento efetivo. Reduções significativas nas mortes por trauma cardíaco poderão ser notadas quando houver integração adequada entre os serviços hospitalares de referência em trauma e o atendimento pré-hospitalar, além das estatísticas do IML. Assim, tornarão mais evidentes as áreas defasadas de atendimento pré-hospitalar, bem como traduzirão às autoridades competentes a importância de se intervir urgentemente nos locais mais violentos ou exigir maior segurança dos veículos automotores. Além disso, a falta de informações, tanto hospitalares quanto médico-legais, em relação ao grau de lesões, prejudica muito a análise comparativa com outros serviços. Torna-se necessário existirem padronizações tanto nos prontuários de atendimento quanto nos atestados de óbito. Otimizar o atendimento, bem como permitir análises críticas da atuação médica na cidade, propiciarão que mais vítimas sobrevivam ao sombrio prognóstico do trauma cardíaco.

\section{AGRADECIMENTOS}

Agradecimento às acadêmicas Gisele Lumy Iguma e Vanessa Ribeiro Santana Berini, da Liga do Trauma da FCM PUC-Campinas, e ao acadêmico Marcello Frederico Santos Schmidt, da Liga do Trauma da FCM-Unicamp, pela efetiva participação e co-autoria no presente estudo.

\begin{abstract}
Background: The vast majority of cardiac trauma victims die before receiving medical care. However, epidemiological studies are few about this injury, describing the patients whom treatment is provided. The objective of this study is to evaluate cardiac injuries victims' profile through the autopsy findings. Methods: We have reviewed 1.976 external causes death cases, which were autopsied in the Campinas Medical Legal Institute, over a two-year period. The cases were assigned for two groups: I, penetrating trauma, and II, blunt trauma. Results: Penetrating trauma was predominant (1.294 cases - 65.5\%). Heart trauma was identified in 359 cases (18.2\%), out of 296 in Group I and 63 in Group II. In Group I, 73.6\% of the victims died at the scene and only 18 patients (6\%) were admitted at hospital and submitted to thoracotomy. In Group II, surgical care was offered for two out of 14 patients (3.2\% of blunt trauma) who were admitted with vital signs. The most affected heart chamber in Group I was the left ventricle (isolated injury in $24.6 \%$ ) and in Group II the was the right ventricle (25\%). Conclusions: We conclude that heart injuries are eminently fatal and only $5.6 \%$ of this victims who died received effective medical care.
\end{abstract}

Key Words: Heart injuries; Heart; Autopsy; Thoracic injuries

\section{REFERÊNCIAS}

1. Crawford Jr FA - “Lesões cardíacas penetrantes”. In Sabiston Tratado de Cirurgia, $15^{\circ}$ Edição. Rio de Janeiro. GuanabaraKoogan, pp. 1819-1823.
2. Ivatury RR - "Injury to the heart". In Mattox KL, Feliciano DV, Moore EE - Trauma. Stanford, Connecticut. Appleton \& Lounge, $3_{\text {th }}$ Edition. 1995, pp. 409-421.

3. Mantovani M, Fraga GP - "Estudo crítico dos óbitos no trauma: experiência da Unicamp”. In Freire, E -Trauma - A doença dos séculos. Rio de Janeiro. Atheneu, 2001, pp. 2851-2861. 
4. Spencer Netto FAC, Campos JM, Lima LFC, et al. - Fatores prognósticos de mortalidade em pacientes com trauma cardíaco que chegam à sala de cirurgia. Rev Col Bras Cir, 2000, 28(1):87-94.

5. Campbell NC, Thomson SR, Muckart DJJ, et al. - Review of 1198 cases of penetrating cardiac trauma. Br J Surg, 1997, 84(12):1737-1740.

6. Kulshrestha P, Das B, Iyer KS, Kumar A, et al. - Cardiac injuries: a clinical and autopsy profile. J Trauma, 1990, 30(2):203-207.

7. Rhee PM, Foy H, Kaufmann C, et al. - Penetrating cardiac injuries: a population-based study. J Trauma, 1998, 45(2):366370.

8. Velmahos GC, Degiannis E, Souter I, et al. - Penetrating trauma to the heart: a relatively innocent injury. Surgery, 1994, 115(6):694-697.

9. Mitchell ME, Muakkassa FF, Poole GV, et al. - Surgical approach of choice for penetrating cardiac wounds. J Trauma, 1993, 34(1):17-20.

10. Burkhart HM, Gomez GA, Jacobson LE, et al. - Fatal blunt aortic injuries: a review of 242 autopsy cases. J Trauma, 2001, 50(1):113-115.

11. Coimbra R, Pinto MC, Razuk A, et al. - Penetrating cardiac wounds: predictive value of trauma índices and the necessity of terminology standardization. Am Surg, 1995, 61(5):448-452.
12. Attar S, Suter CM, Hankins JR, et al. - Penetrating cardiac injuries. Ann Thorac Surg, 1991, 51(5):711-716.

13. Henderson VJ, Smith RS, Fry WR, et al. - Cardiac injuries: analysis of an unselected series of 251 cases. J Trauma, 1994, 36(3):341-348.

14. Kavolius J, Golocovsky M, Champion HR - Predictors to outcome in patients who have sustained trauma and who undergo emergency thoracotomy. Arch Surg, 1993, 128(10):1158-1162.

15. Baccarin V, Rizoli SB, Vieira RW, et al. - Ferimentos cardíacos penetrantes: experiência de 21 casos. Rev Col Bras Cir, 1992, 19(3):112-115.

16. Durham LA, Richardson RJ, Wall MJ, et al. - Emergency center thoracotomy:impact of prehospital resuscitation. J Trauma, 1992, 32(6):775-779.

Endereço para correspondência:

Gustavo Pereira Fraga

Av. Coronel Silva Telles, 211 apto 3

13024-000 Cambuí Campinas-SP

fragagp@uol.com.br 